\title{
DIAGNOSTIC THORACOTOMY FOR SOLID PULMONARY INFILTRATES
}

\author{
BY \\ E. HUSFELDT AND C. J. CARLSEN \\ From the Department of Thoracic Surgery, University Hospital, Copenhagen
}

The progress of thoracic surgery within the last decade has solved many important problems, but it has also posed others. One of these is the correct attitude towards that group of patients who previously were kept under observation, namely, those who exhibit a round, sharply demarcated, solid shadow in the lung field as a chance finding (for example, in a routine radiograph or at massradiography) without subjective or objective symptoms. This study does not include cases in which the radiological appearances suggested lesions issuing from the mediastinum or from the chest wall. These cases have been well known for a long time, but they are now being detected in larger numbers because of the increased use of chest radiography and more particularly of mass radiography.

With the diagnostic means available to-day it is possible to make an exact diagnosis in almost every case of haemoptysis, persistent cough, dyspnoea, thoracic pain, but in most of these symptomless cases it is impossible to decide the nature of the disease, even when all modern diagnostic means are utilized. For these reasons it was decided, in 1942, to operate in such cases unless there were serious contraindications to surgery.

Within other fields of surgery exploratory interventions are accepted in obscure cases. In our opinion, the risk involved by exploratory thoracotomy is slight in view of the present stage of operative technique, anaesthesia, and pre- and postoperative treatment. In addition, these patients stand exploratory thoracotomy much better than patients with clinically manifest malignancies.

This series of 33 patients is "selected" because it includes patients who have entered the hospital after a long or short period of observation elsewhere, usually because they have been suspected of malignancy on the basis of the radiological findings and their age. This study affords no data as to the incidence of the condition among the population as a whole and no conclusion as to the procedure of choice in "young" patients, that is, those under about 35 years of age.

It is common to all the patients that they exhibited a pulmonary shadow of the kind described above, had no symptoms, and failed to show any other signs of diagnostic value after Wassermann tests, examinations of the sputum for tubercle bacilli and tumour cells, bronchoscopy, and extended radiological examinations.

The sex and age distribution as well as the post-operative diagnosis are tabulated.

At the present time it may be said that our principle of treatment was justified by demonstrating four early cases of carcinoma, and these were considered to have a better prognosis than patients who underwent operation after symptoms had 
TABLE

Sex, Age Distribution, and Post-operative Diagnosis of 33 Cases

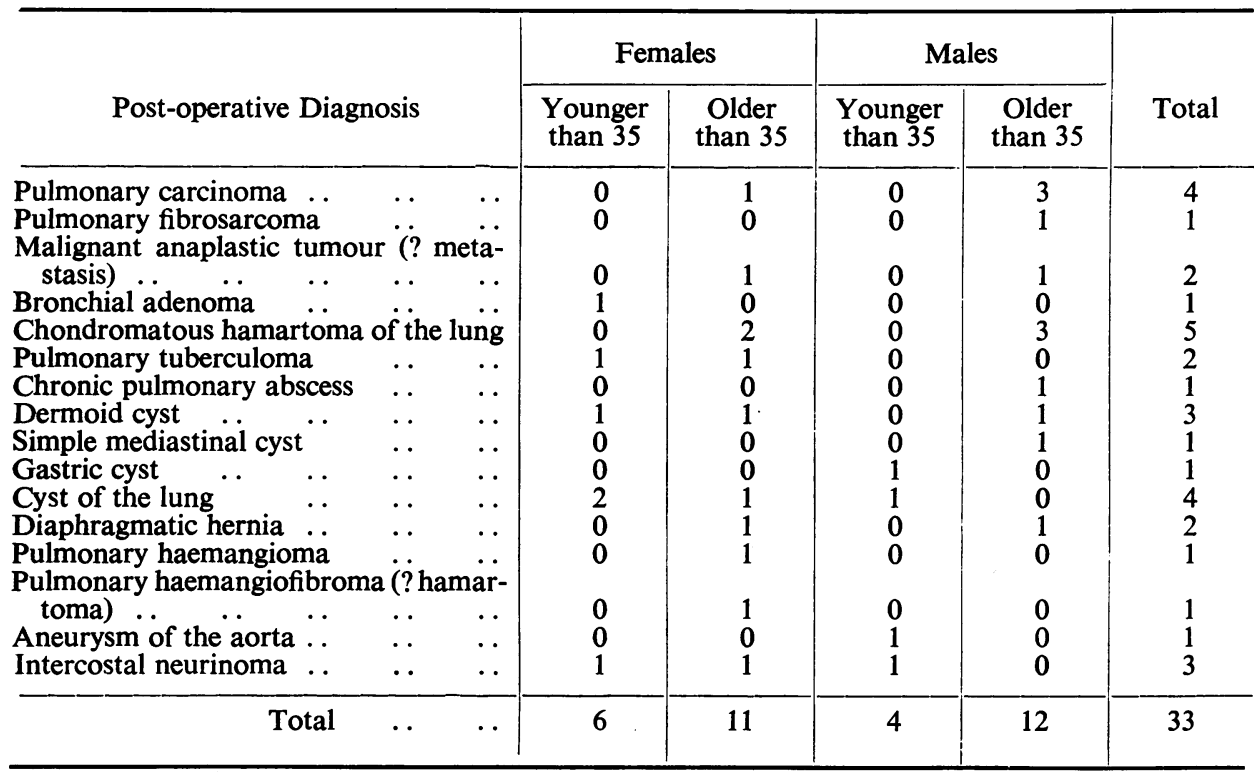

become manifest. This group included a woman, aged 57, who had been observed for 27 months during which period the shadow had remained unchanged. In the remaining three, the observation period had ranged from two and a half to 14 months, and the shadows had remained unchanged.

In the case of fibrosarcoma, a 67-year-old man, the shadow had remained unchanged for five months, when he was referred for operation.

A 60-year-old woman was proved to be suffering from an anaplastic malignant tumour (? metastasis). The shadow had remained unchanged for two years, but then it increased somewhat in size, and the patient was sent to us.

The other patient in this group was a man, aged 52, who was admitted to hospital because the shadow had increased slightly after having been stationary for four months.

In the case of bronchial adenoma (a woman, aged 32) the shadow had remained unchanged for two months. According to recent experiences, it is agreed that bronchial adenomas should be regarded and treated as potentially malignant growths.

The five cases of chondromatous hamartoma of the lung will be reported in a communication (to be published) by Carlsen and Kiaer. They recommend operation because the lesion cannot be differentiated from carcinoma without operation, and also because of the theoretical possibility that malignant change may have occurred, although definite instances of malignant degeneration of hamartoma have not been reported so far.

It is often difficult to make a clinical or radiological diagnosis of tuberculoma of the lung. Tomography, which should be done in cases of this nature, may reveal cavitation. But the latter may occur in cases of small carcinomas as well as of 
chondromatous hamartomas and thus is of limited diagnostic value. In addition, it may happen that operation, as in one of our cases, reveals fluid in a tuberculoma which has appeared solid on the tomogram.

Too little is known about the further course of tuberculomas, but in our opinion, operation should be done in the presence of a pre-operative diagnosis or likelihood of tuberculoma. Re-examination of our cases did not show spread of the tuberculosis or other complications in any of our patients.

A chronic abscess of the lung must be treated surgically, since it will otherwise give rise to secondary bronchiectases or acute complications.

A diagnosis of dermoid cyst can often be made by radiographs, but in these three cases, the appearances suggested solid lung tumours. Unoperated dermoid cysts of the chest usually become locally malignant.

The gastric cyst occurred in a boy of 22 months. The history of this boy has previously been reported by Christoffersen (1948), who emphasized that most of the reported cases had been diagnosed post mortem, perforation of the cyst being the cause of death.

As a rule, simple mediastinal cysts can be diagnosed by radiographs, but in our case the cyst simulated a solid tumour. The same applies to the four cysts of the lung, for which the operative indications have been dealt with, amongst others, by Wainö Andersen (1947) in a report on the experience gained in this department.

The radiological diagnosis of diaphragmatic hernia is usually easy, but hernia through Morgagni's foramen often constitutes an exception, being in most cases filled with omental fatty tissue and simulating a solid growth. The patient with hernia through Morgagni's foramen was a woman aged 44, who had been observed for 11 years during which period the shadow had remained unchanged. Thereupon it began increasing in size and the patient entered our hospital. The other patient in this group was a man aged 55, and radiographs of his chest suggested hernia, but this diagnosis was abandoned after a radiological examination of the stomach had shown normal conditions.

The patient with pulmonary haemangioma, a 55-year-old woman, reported with a history of " haematemesis" 25 years previously, for which she had received medical management with good result. One month before admission there had been a small " haematemesis." In the patient's opinion, she had not coughed the blood up. During her stay in hospital blood was not found in either sputum or vomitus. A woman, aged 58, proved to be suffering from " a haemangiofibromatous tumour," possibly a " hamartoma " of a type different from the chondromata. It had remained stationary for eight months. It is reasonable to presume that these two patients would have run a considerable risk of haemoptyses if no operation had jeen done.

In the case of a man, aged 31 and observed for 20 months, operation unexpectedly revealed a thin-walled aneurysm of the descending aorta. Its wall was so thin that the blood could be seen passing through it ; the Wassermann reaction was negative, and the lesion was probably congenital. Judging by its appearance, the lesion had to be considered a grave risk.

A pre-operative diagnosis could not be made in the three patients suffering from intercostal neurinoma. In a certain number of cases, these growths undergo malignant degeneration, and they may precipitate destruction of the adjacent ribs. In our opinion they should be removed surgically. 
On the whole, it seems justifiable to conclude that much has been gained by the principle of performing thoracotomy in these cases. Only one patient died after the operation, namely, the patient with aneurysm of the aorta who succumbed to renal insufficiency and ischaemic necrosis of the spinal cord 18 days after local resection and anastomosis of the aorta.

\section{SUMMARY}

From 1942 thoracotomy has been the routine procedure of the Department of Thoracic Surgery of the University Hospital, Copenhagen, in cases exhibiting round, sharply demarcated, solid shadows in the lungs without other symptoms. The series comprises a total of 33 patients, "selected" in the sense that 23 were over 35 years of age. The operative findings included four small, sharply demarcated primary carcinomas.

\section{REFERENCES}

Andersen, E. Wainö (1947). Nord. Med., 35, 1703.

Carlsen, C. J., and Kiaer, W. (1950). Chondromatous Hamartoma of the Lung. To be published. Christoffersen, J. V. (1948). Acta chir. scand., 96, 12. 